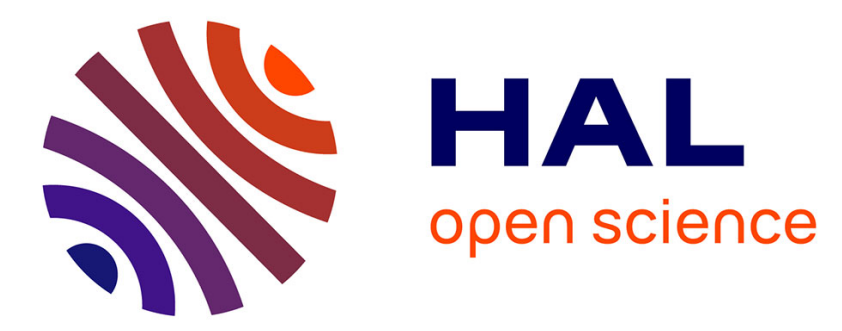

\title{
Gravure hélicon de l'InP en plasma HBr. Morphologie et caractérisation des défauts de surface
}

\author{
J. Etrillard, P. Ossart, G. Patriarche, J. Francou
}

\section{To cite this version:}

J. Etrillard, P. Ossart, G. Patriarche, J. Francou. Gravure hélicon de l'InP en plasma HBr. Morphologie et caractérisation des défauts de surface. Journal de Physique III, 1995, 5 (5), pp.467-481. 10.1051/jp3:1995140 . jpa-00249324

\section{HAL Id: jpa-00249324 https://hal.science/jpa-00249324}

Submitted on 1 Jan 1995

HAL is a multi-disciplinary open access archive for the deposit and dissemination of scientific research documents, whether they are published or not. The documents may come from teaching and research institutions in France or abroad, or from public or private research centers.
L'archive ouverte pluridisciplinaire HAL, est destinée au dépôt et à la diffusion de documents scientifiques de niveau recherche, publiés ou non, émanant des établissements d'enseignement et de recherche français ou étrangers, des laboratoires publics ou privés. 
Classification

Physics Abstracts

$52.40 \mathrm{H}-52.70-52.75-73.20$

\title{
Gravure hélicon de l'InP en plasma HBr. Morphologie et caractérisation des défauts de surface
}

J. Etrillard $\left({ }^{1}\right)$, P. Ossart $\left({ }^{1}\right)$, G. Patriarche $\left({ }^{2}\right)$ et J.M. Francou $\left({ }^{3}\right)$

( ${ }^{1}$ ) France Telecom/CNET/PAB, Laboratoire de Bagneux, 196 rue Henri Ravera, BP. 107, 92220 Bagneux Cedex, France

$\left({ }^{2}\right)$ Institut des Matériaux de Nantes, 2 rue de la Houssinière, 44072 Nantes, France

$\left({ }^{3}\right)$ France Telecom, CNET CNS, 38 chemin du vieux chêne, 38243 Meylan, France

(Reçu le 8 juillet 1994, révisé le 10 novembre 1994, accepté le 18 novembre 1994)

Résumé. - Nous exposons les résultats de l'étude de la gravure en plasma HBr de l'InP en décharge hélicon en fonction de la température du substrat, de l'énergie ionique, de la pression de travail et de la puissance de source. La morphologie de surface ainsi que les profils de gravure sont examinés par microscopie à balayage. Les défauts de flancs sont mis en évidence par microscopie à transmission dans le cas de gravures anisotropes de motifs submicroniques. L'analyse Auger ainsi que des mesures de photoluminescence ont été utilisées pour évaluer la pollution, les désorptions sur une profondeur de $20 \mathrm{~nm}$ ainsi que les défauts électriques résultants. Des gravures anisotropes à faibles dommages induits ont été obtenues avec des vitesses de gravure très acceptables. Cependant une importante amorphisation des flancs a été mise en évidence.

\begin{abstract}
We report the results of InP etching using a helicon discharge plasma. The etched surface morphology and the pattern profiles were examined by scanning electron microscopy (SEM). Transmission electron microscopy (TEM) was used to observe the sidewall damage for anisotropic etching. Auger electron spectroscopy (AES) was used to obtain the elemental composition in the top $20 \mathrm{~nm}$ of the etched surfaces and to evaluate the contamination and desorption. We have also characterized surface and subsurface damage in InP etched substrates using photoluminescence intensity. Anisotropic submicronic etched patterns with low damage were obtained with high etch rates. However, a significant amorphisation of the sidewalls have been evidenced by TEM.
\end{abstract}

\section{Introduction}

L'utilisation généralisée des techniques de gravure sèche en microélectronique répond à un besoin croissant d'augmentation des densités d'intégration. Les techniques de gravure ionique réactive (RIE) très largement utilisées, tant en production qu'en recherche, montrent désormais leurs limites dans le domaine des défauts qu'elles créent en surface [1]. De nombreuses recherches visent actuellement à mettre au point de nouvelles sources de gravure moins agressives vis 
à vis des matériaux fragiles que constituent les matériaux III-V par exemple. De nouveaux procédés de gravure réactive [2] utilisant ces nouvelles sources ont précédé, en production de composants silicium, une compréhension plus approfondie des phénomènes originaux relatifs à ces techniques [3].

Les sources ECR ont suscité la plus grande part des efforts menés dans ce domaine des gravures à faibles dommages induits [4]. Cependant, les sources à couplage inductif telles que l'hélicon font l'objet d'un intérêt grandissant. Ces sources sont constituées d'une antenne externe alimentée par un signal radio-fréquence à $13.56 \mathrm{MHz}$. L'onde TEM résultante se propage dans un champ magnétique longitudinal constant et donne naissance à un mode de propagation hélicon. Ce type de décharge permet de produire des plasmas de haute densité ionique indépendamment de l'énergie des ions réactifs dont on sait le rôle prédominant dans la création et l'amplitude des défauts induits en RIE [5]. Une polarisation, indépendante de la source, permet d'accélérer les ions vers le substrat avec une énergie aussi faible que possible.

\section{Expérimentation}

Dans cette étude, nous avons évalué les caractéristiques de gravure de l'InP par ce mode de gravure dans le bâti RIPE 160 d'Alcatel. Ce bâti est alimenté par une source constituée d'une antenne dont la puissance est réglable de 0 à $2 \mathrm{~kW}$. La propagation hélicoïdale a lieu grâce à un champ magnétique constant de 100 Gauss. Le réacteur permet la gravure d'un échantillon de 4 pouces de diamètre refroidi par un flux d'hélium liquide. La température est réglable de $-100^{\circ} \mathrm{C}$ à $+40^{\circ} \mathrm{C}$. Le chargement est effectué par sas évitant une longue procédure de refroidissement avant ouverture. Le substrat peut être polarisé entre $20 \mathrm{~V}$ et $130 \mathrm{~V}$. Le plasma de diffusion est confiné par un champ permanent fourni par huit aimants répartis uniformément autour de la chambre.

Par ailleurs nous avons utilisé une chimie à base de brome [6] qui fournit des composés d'indium et de phosphore de pression de vapeur comparables à celles des composés chlorés et qui ne présentent pas l'inconvénient de formation de polymères des plasmas $\mathrm{CH}_{4}-\mathrm{H}_{2}$. Nous nous sommes attachés à caractériser la morphologie de gravure par microscopie électronique en fonction de l'énergie ionique en limitant celle-ci à $130 \mathrm{eV}$. Une étude a également été menée en fonction de la pression de la décharge ainsi qu'en fonction de la température du substrat qui joue, entre autres, un rôle déterminant sur la morphologie de surface.

Les substrats utilisés ont été masqués par de la résine photosensible ou du platine. Les motifs lithographiés sont constitués de lignes de 1 à quelques microns de largeur ou de petits plots circulaires de quelques fractions de micron de diamètre.

Une étude de la propreté de surface a été menée par spectroscopie Auger. Les défauts électriques ont été caractérisés par photoluminescence. Les profils de gravure ont été observés par microscopie électronique à balayage et à transmission. Les vitesses d'attaque ont été déterminées par mesure de marche par profilomètre.

\section{Résultats}

2.1. Vitesse DE GRAVURE ET MORPhOlOGIE DE SURFACE. - Les vitesses d'attaque en fonction des différents paramètres de gravure ainsi que la morphologie de surface après gravure sont présentées sur les figures 1 à 6 . On constate, sur la figure 1 une indépendance de la vitesse avec la température régulée au niveau du substrat qu'on supposera, dans la suite de cet exposé, sensiblement égale à celle du substrat (bien que la température de surface de l'échantillon soit probablement beaucoup plus élevée). Cette indépendance de la vitesse avec la température traduit la faible évolution de la pression de vapeur saturante des composés bromés de l'indium, 


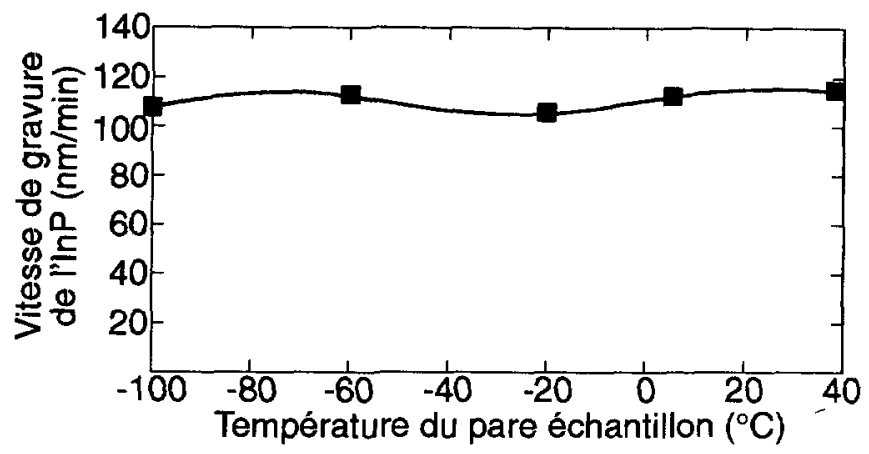

Fig. 1. - Variation de la vitesse de gravure de l'InP en fonction de la température du substrat. Les échantillons ont été gravés avec $25 \mathrm{sccm}$ de $\mathrm{HBr}, 1,5 \mathrm{~kW}$ de puissance source, à $1,5 \mathrm{~m}$ Torr de pression totale. La polarisation est de $-100 \mathrm{~V}$.

[Dependence of InP etch rate on chuck temperature. Samples were etched with $25 \mathrm{sccm} \mathrm{HBr}, 1.5 \mathrm{~kW}$ source power at $1.5 \mathrm{~m}$ Torr. Bias voltage was $-100 \mathrm{~V}$.]

$\mathrm{InBr}_{3}$ principalement, beaucoup plus faible que la pression de travail dans cette gamme de température [7]. Concernant l'indium, la gravure est principalement due à la pulvérisation simple des atomes ceci dans l'hypothèse d'une faible différence entre la température globale du substrat et la température de surface. Concernant le phosphore, la forte pression de vapeur de la phosphine permet de penser qu'en plus de la gravure par pulvérisation cathodique possible s'ajoute une gravure réactive. La figure 2 montre que la surface après gravure reste très lisse pour des températures basses du substrat alors qu'une dégradation importante est constatée pour une température de $+40^{\circ} \mathrm{C}$. Il y a dégradation progressive de la rugosité avec l'élévation de température. Un effet important de tranchées (trenching effect), également significatif de la pulvérisation cathodique, est observé figure 2 sur les motifs gravés aux températures supérieures $\grave{a}-60^{\circ} \mathrm{C}$. Ce phénomène peut être expliqué par une modification de la résistance de couche (sheet resistance) du matériau [8] évoluant fortement, suivant la température de la surface pendant la gravure, à cause des désorptions de phosphore.

Les vitesses sont, par contre, fortement variables avec l'énergie ionique (considérée ici comme sensiblement égale à l'énergie potentielle déterminée par la polarisation du substrat), comme en témoigne la figure 3. L'évolution est d'abord linéaire puis, au delà de $60 \mathrm{eV}$ devient exponentielle. Cette évolution doit être corrélée à l'évolution, illustrée sur la figure 4, de la vitesse de gravure en fonction du courant ionique reçu par l'échantillon pour les mêmes conditions expérimentales que celles utilisées en figure 3. La vitesse de gravure est sensiblement proportionnelle au courant d'ions arrivant à la surface de l'échantillon pour des valeurs de courant inférieures à $55 \mathrm{~mA} / \mathrm{cm}^{2}$ ou à $50 \mathrm{~mA} / \mathrm{cm}^{2}$ lorsque les ions ont une énergie de $100 \mathrm{eV}$ ou de $60 \mathrm{eV}$ respectivement. Puis, la vitesse de gravure augmente avec une pente plus grande au delà de ces valeurs du courant.

Par conséquent, on peut affirmer que la vitesse de gravure est à la fois proportionnelle à l'énergie des ions mais aussi à leur nombre, c'est à dire en fait à la puissance incidente transférée à l'échantillon. Ces résultats sont cohérents avec la théorie de la pulvérisation cathodique établie, dans le cas d'ions de gaz inertes sur des matériaux monoatomiques [9]. Cependant, cette conclusion n'est valable qu'en deçà d'un certain seuil. Ce seuil de la valeur de la puissance incidente est fonction de l'énergie des ions : il est de $3 \mathrm{~W} / \mathrm{cm}^{2}$ pour des ions de $60 \mathrm{eV}$ et de $5.5 \mathrm{~W} / \mathrm{cm}^{2}$ lorsque l'énergie ionique est portée à $100 \mathrm{eV}$. 


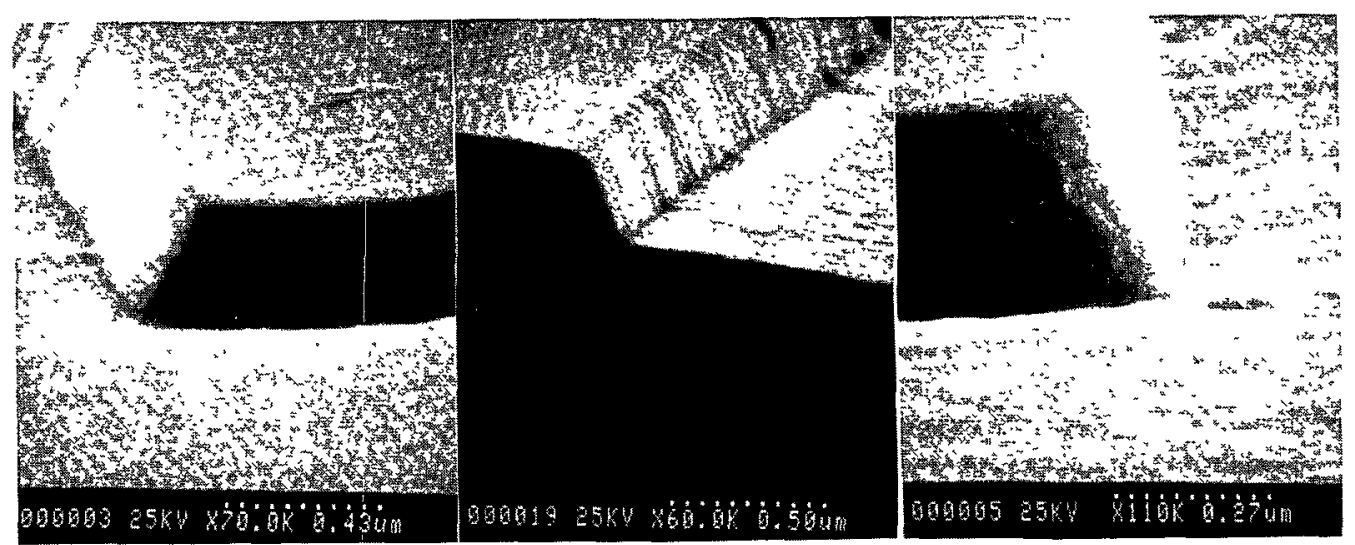

- I0Regres

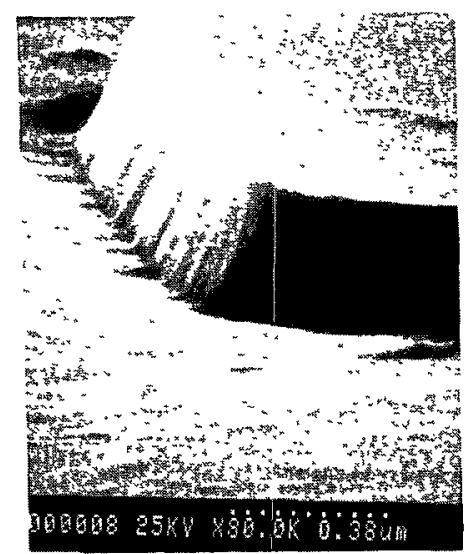

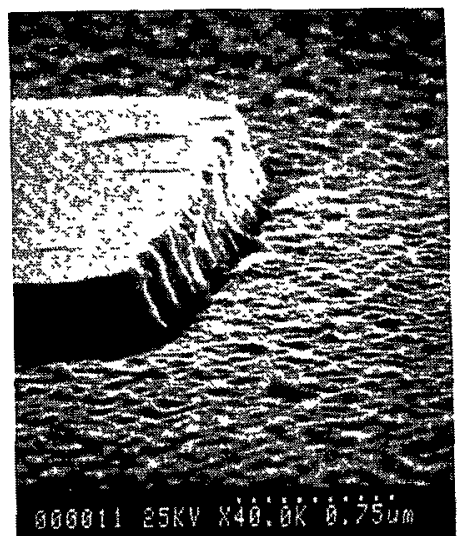

440. degres

Fig. 2. - Influence de la température du substrat sur le "trenching effect". Aucun effet n'est constaté en deçà de $-60^{\circ} \mathrm{C}$.

[Dependence of trenching effect on the chuck temperature. No trenching occurs for low temperature etching. Samples were etched with the process parameters described in Figure 1.]

Au delà de ces valeurs de la densité de puissance, la vitesse de gravure suit une courbe exponentielle.

Ces résultats peuvent suggérer qu'à une gravure assistée par les ions c'est à dire conduisant à une vitesse proportionnelle à leur nombre, s'ajoute, au delà du seuil précédemment défini, une désorption spontanée, probablement du phosphore, provoquée par l'élévation de la température de surface. Ce départ de phosphore augmente le taux de pulvérisation cathodique de l'indium. Ce taux est, en effet, inversement proportionnel à l'énergie de liaison à la surface du matériau. On peut également penser que, pour une même température du substrat, la proportion de la 


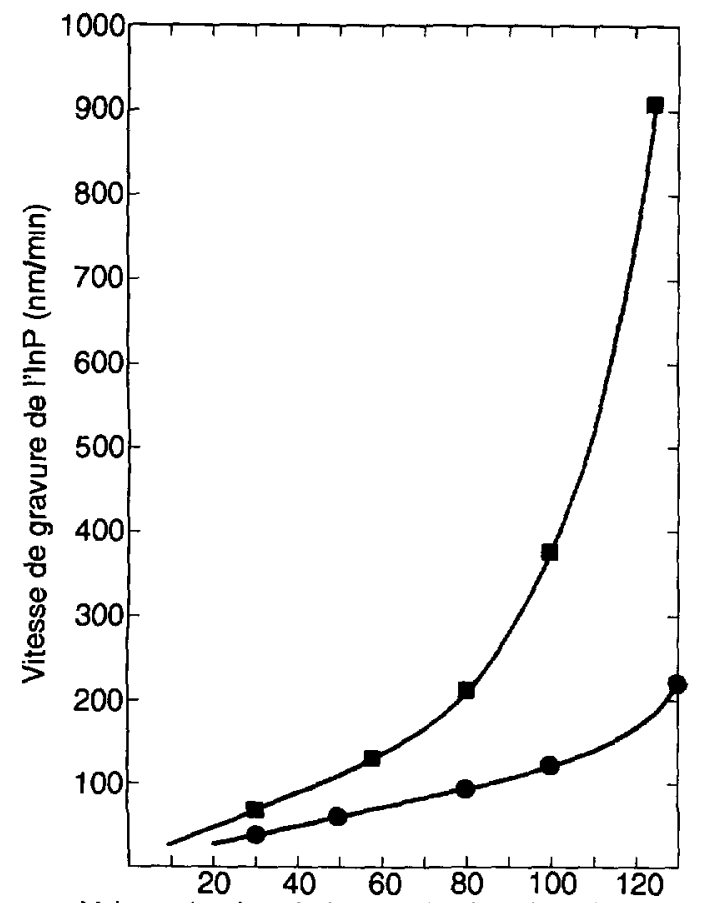

Valeur absolue de la polarisation du substrat (V)

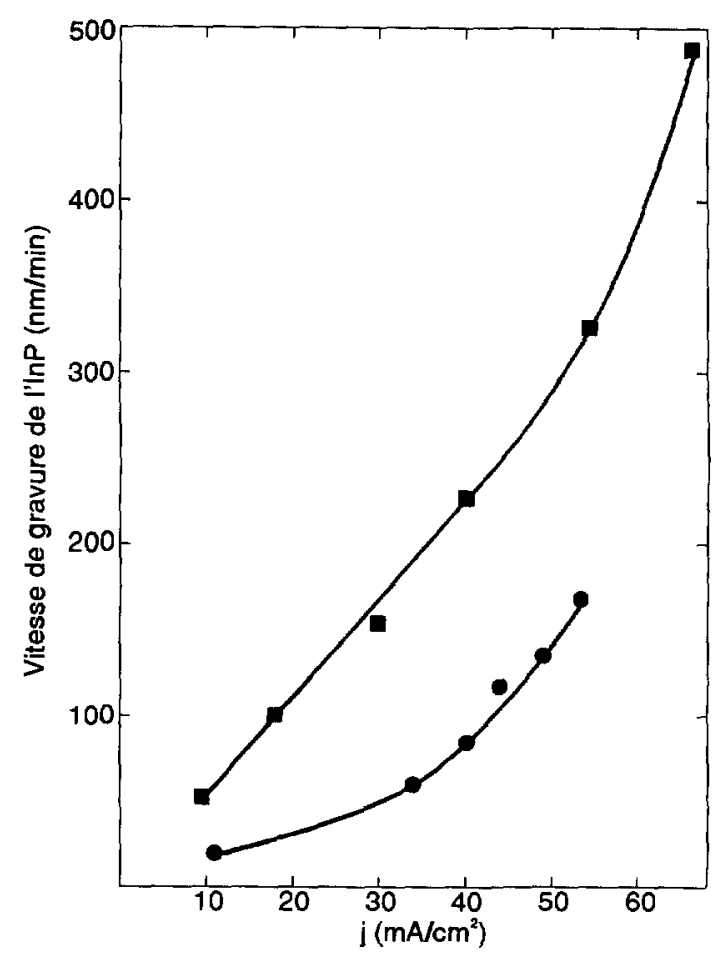

Fig. 4

Fig. 3

Fig. 3. - Variation de la vitesse de gravure en fonction de la tension de polarisation du substrat. Les échantillons sont gravés avec $25 \mathrm{sccm}$ de $\mathrm{HBr}, 1,5 \mathrm{~kW}$ de puissance source, à $1,5 \mathrm{~m}$ Torr (•) et $0,38 \mathrm{~m}$ Torr ( $\mathbf{\square}$ ).

[Dependence of InP etch rate on bias voltage. Samples were etched with $25 \mathrm{sccm} \mathrm{HBr}, 1.5 \mathrm{~kW}$ source power, at $1.5 \mathrm{~m}$ Torr $(\bullet)$ and $0.38 \mathrm{~m}$ Torr $(\boldsymbol{D})$.]

Fig. 4. - Variation de la vitesse de gravure de l'InP en fonction de la densité du courant d'ions incidents. Les échantillons sont gravés à $-60 \mathrm{~V}(\bullet)$ et $-100 \mathrm{~V}(\mathbf{\square})$.

[Dependence of InP etch rate on ion current density. Samples were etched with $10 \mathrm{sccm} \mathrm{HBr}$ at bias voltage of $-60 \mathrm{~V}(\bullet)$ and $-100 \mathrm{~V}(\mathbf{a}) .1$

gravure par désorption spontanée par rapport à la gravure assistée par les ions, est plus forte à basse énergie ionique puisque les désorptions spontanées ne dépendent que de la température. Cette hypothèse expliquerait que le seuil de densité de puissance soit fonction de l'énergie ionique et, qu'en l'occurence, il soit plus faible à basse énergie ionique. Il faut admettre, dans ce cas, que la température de surface soit plus importante que $40^{\circ} \mathrm{C}$, température maximale régulée dans l'étude de l'influence de la température illustrée par la figure 1.

La figure 5 illustre les variations de la vitesse de gravure avec la pression totale du plasma. On observe une forte décroissance expliquée par la plus faible longueur de diffusion des ions actifs depuis la source jusqu'au substrat.

L'évolution de la vitesse de gravure avec la puissance de source est observée sur la figure 6. 


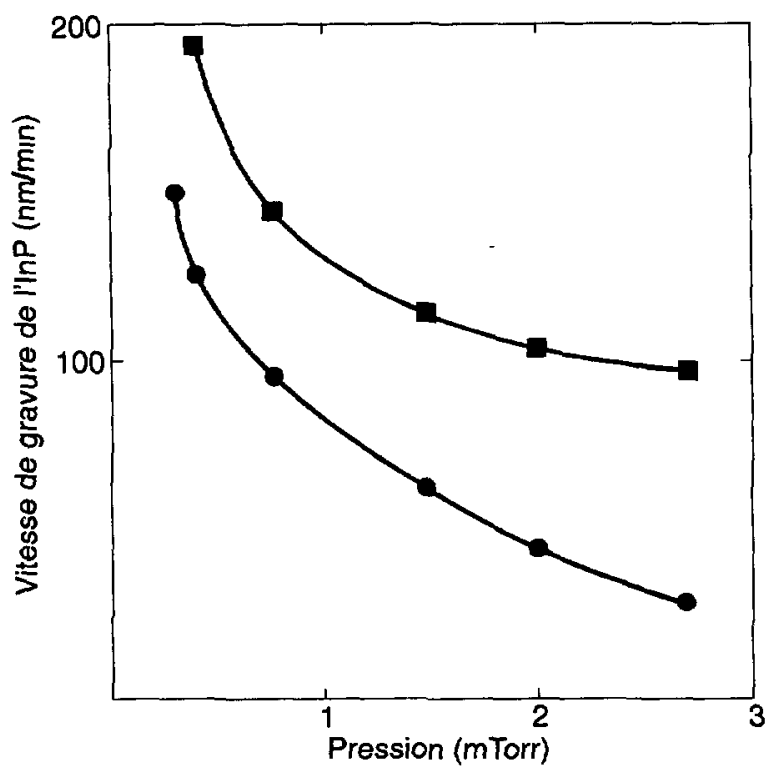

Fig. 5

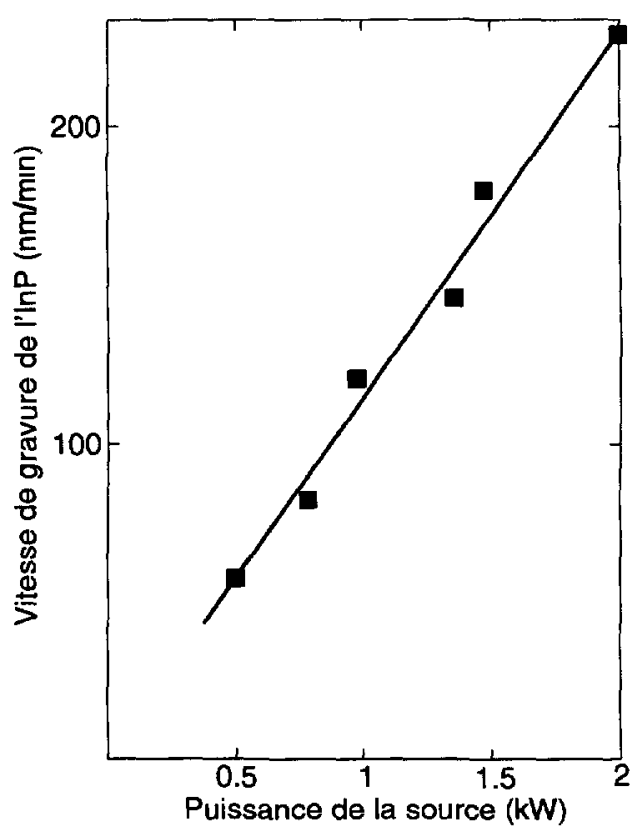

Fig. 6

Fig. 5. - Variation de la vitesse de gravure en fonction de la pression du plasma. Le débit est de 10 sccm de $\mathrm{HBr}$ à $1,5 \mathrm{~kW}$ de puissance source, avec des polarisations de $-60 \mathrm{~V}(\bullet)$ et $-80 \mathrm{~V}(\boldsymbol{\square})$.

[Dependence of InP etch rate on plasma pressure. Samples were etched with $10 \mathrm{sccm} \mathrm{HBr}$ at $1.5 \mathrm{~kW}$ source power and $-60 \mathrm{~V}$ bias $(\bullet)$ and $-80 \mathrm{~V}(\mathbf{\square})$.]

Fig. 6. - Variation de la vitesse de gravure en fonction de la puissance de la source hélicon. La pression est de $1,5 \mathrm{~m}$ Torr, le débit de $25 \mathrm{sccm}$ et la polarisation de $-125 \mathrm{~V}$.

[Dependence of InP etch rate on power source. Samples were etched at $1.5 \mathrm{~m}$ Torr, $25 \mathrm{sccm} \mathrm{HBr}$ and a bias voltage of $-125 \mathrm{~V}$.]

Cette courbe est linéaire témoignant d'une évolution également linéaire de la densité ionique avec la puissance source.

2.2. Profils De gravure et SÉLECTIVItÉ. - Les profils de gravure ont été observés par microscopie électronique ce qui a permis de caractériser simultanément l'état de surface après gravure ainsi que l'anisotropie de gravure.

Les effets de trenching ainsi que la rugosité observés lors des gravures à température supérieure à $-60^{\circ} \mathrm{C}$ ont été décrits sur la figure 2 dans la section précédente. Pour s'affranchir de ces phénomènes, les études de sélectivité ont été menées à une température de $-60^{\circ} \mathrm{C}$. La sélectivité vis à vis de la résine en fonction de l'énergie ionique est illustrée sur la figure 7 . Pour les valeurs inférieures à $60 \mathrm{eV}$, on obtient une valeur maximale de 1,3 . Cette valeur est bien insuffisante pour éviter les transferts de pente entre le masque de résine et l'InP gravé. Cette situation est visible sur la figure 8 sur laquelle on observe le profil en pente du masque de résine avant gravure puis le motif gravé dans l'InP surmonté du masque de résine.

La très faible valeur de la sélectivité de gravure de l'InP vis à vis de la résine, ne permet pas d'étudier l'anisotropie de gravure à cause des profils des masques et du transfert de pente qui en résulte comme l'illustre la figure 8. Le rôle de l'effet facette (faceting effect) caractéristique de la 


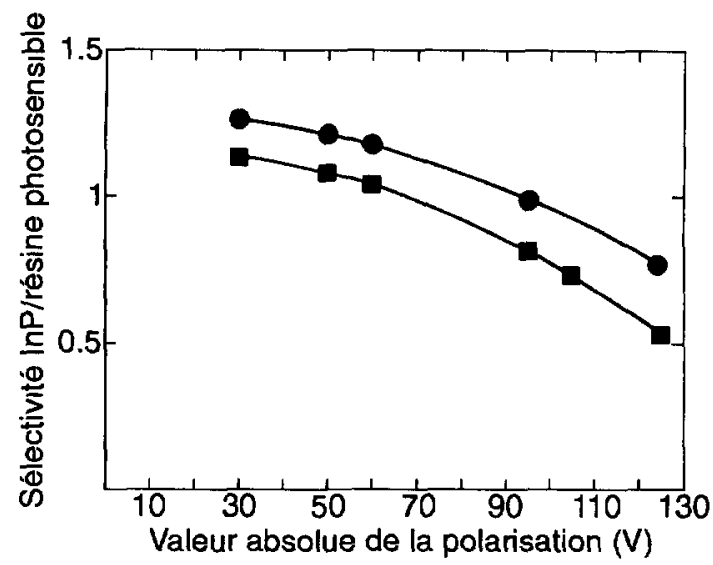

Fig. 7. - Sélectivité vis à vis de la résine du plasma de gravure en fonction de la tension de polarisation. Les échantillons ont été gravés avec $10 \mathrm{sccm}$ de $\mathrm{HBr}, 1,5 \mathrm{~kW}$ de puissance source, à $1,5 \mathrm{~m}$ Torr ( $)$ et $0,4 \mathrm{~m}$ Torr (ם).

[The InP to resist selectivity is shown as a function of bias voltage. Samples were etched with $10 \mathrm{sccm}$ $\mathrm{HBr}, 1.5 \mathrm{~kW}$ source power at $1.5 \mathrm{~m}$ Torr $(\bullet)$ and $0.4 \mathrm{~m}$ Torr (ם).]

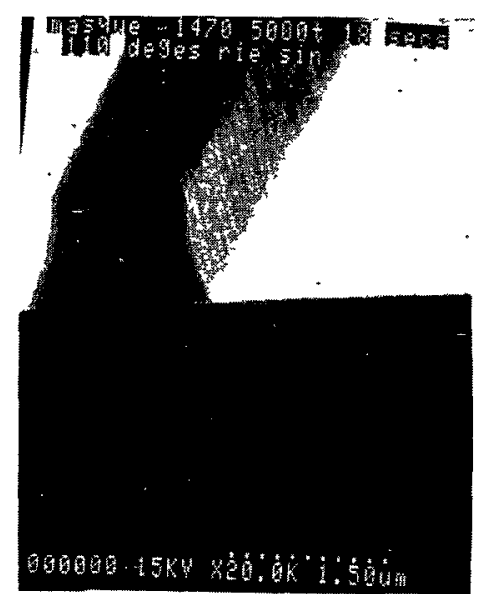

a)

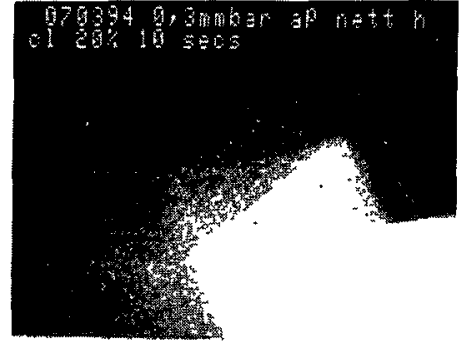

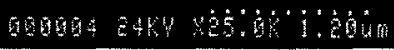

b)

Fig. 8. - Illustration du transfert de pente entre le masque de résine et le substrat InP. Le masque de résine est observé avant gravure en $8 \mathrm{a}$. Le transfert de pente est visible en $8 \mathrm{~b}$. L'effet de facette amplifie le phénomène.

[A significant slope transfer is observed due to the low value of the resist to InP selectivity. In Figure 8a the cross-section of the resist mask is shown. In Figure $8 \mathrm{~b}$ the etched sample cross-section demonstrate the slope transfer which is reinforced by a faceting effect on the resist mask.]

pulvérisation cathodique est important dans ce transfert comme on peut également l'observer sur la figure 8. Les ions ne sont pas très énergétiques mais leur nombre est très grand. Cependant plus l'énergie ionique est proche du seuil de pulvérisation et moins le rendement de pulvérisation est élevé. C'est ce qui explique l'apparente meilleure directivité de gravure à basse énergie ionique. Aucune gravure anisotrope n'a pu être obtenue dans l'étude paramètrique entreprise 
avec ce type de masque de résine à parois obliques. Il est probable qu'une anisotropie correcte puisse être obtenue avec un masque de résine à parois verticales malgré le manque de sélectivité à condition que ce masque soit suffisamment épais.

Une anisotropie très correcte peut être obtenue avec un masque en platine avec lequel la sélectivité est au minimum 20 fois supérieure à celle de la résine. Cependant, là encore, les effets de pulvérisation cathodique peuvent diminuer l'anisotropie apparente des gravures. La figure 9 montre un réseau de motifs gravés dans InP masqués par des plots circulaires de platine d'un diamètre de $0,15 \mu \mathrm{m}$. L'énergie ionique utilisée pour obtenir cette directivité de gravure a été de $125 \mathrm{eV}$. Le facteur de forme est d'environ 14 . Il faut cependant noter que le masque a complètement disparu sur ces motifs par pulvérisation cathodique du platine causée par les ions positifs du plasma. On remarque aussi la rugosité de la surface témoignant de la présence de défauts induits par les ions.

2.3. Composition de surface. - Les analyses des surfaces menées par spectroscopie Auger après gravure sont relatives à la composition de surface ainsi qu'à la composition des couches immédiatement sous jacentes jusqu'à une profondeur de $20 \mathrm{~nm}$ environ. Nous exposons d'abord les résultats relatifs à la composition de surface.

Concernant la composition de surface, les principaux résultats sont les suivants :

a) On observe une faible concentration atomique de phosphore en surface illustrée sur la figure 10. Ce phénomène est d'autant plus important que l'énergie ionique est élevée. Il y a, par exemple, une concentration atomique de surface de $4 \%$ du phosphore lorsque l'énergie ionique est de $125 \mathrm{eV}$ et de $21 \%$ avec une énergie de $30 \mathrm{eV}$. La courbe relative au rapport des concentrations de surface d'indium et de phosphore montre que le départ préférentiel du phosphore est amplifié quand l'énergie ionique croit. A plus haute température le déséquilibre évolue peu avec l'énergie ionique. Cependant, pour une énergie ionique de $30 \mathrm{eV}$, ce rapport des concentrations de l'indium par rapport au phosphore est plus élevé quand la température du substrat augmente $\left(3,8\right.$ à $25^{\circ} \mathrm{C}$ au lieu de 2,0 à la température de $-60^{\circ} \mathrm{C}$ ). Cette dégradation de la stœchiométrie de surface ne permet cependant pas de séparer les influences respectives de la température et de l'énergie ionique.

b) Le départ préférentiel de surface du phosphore est aussi d'autant plus important que la température du substrat, pendant la gravure, est élevée : ce départ atteint $41 \%$ pour une température de gravure de $25^{\circ} \mathrm{C}$ et seulement $29 \%$ si la température est de $-60^{\circ} \mathrm{C}$, ceci pour une même énergie ionique de $30 \mathrm{eV}$ (Fig. 10).

c) Les surfaces sont polluées essentiellement par le carbone et l'oxygène. Aucune trace de brome n'est relevée malgré les faibles températures étudiées dans une partie des expériences. La figure 11 montre l'évolution de la concentration de surface en oxygène et carbone en fonction de l'énergie ionique utilisée pour la gravure. La pollution de surface croit avec l'énergie ionique. La température influe également sur la pollution de surface qui est, à énergie ionique constante, plus faible quand la gravure est effectuée à basse température. On peut penser que la pollution en carbone et oxygène est d'autant plus importante que les défauts structurels induits sont nombreux. Ces défauts favorisent l'oxydation de surface et la contamination par le carbone.

Une étude a également été menée sur la composition en profondeur des matériaux gravés. La figure 12 montre la profondeur sur laquelle le départ préférentiel en phosphore est constaté. Au delà de cette profondeur on retrouve la stœchiométrie originelle du matériau et aucune pollution 